\title{
Agentes Conversacionais no Ensino-Aprendizagem de Línguas
}

\author{
José Paulo de Araújo \\ UFRJ
}

\begin{abstract}
Resumo:
Este artigo apresenta uma revisão da literatura sobre o uso de agentes conversacionais (chatbots) AIML no apoio aos processos de ensino e aprendizagem de língua estrangeira/segunda língua. Defende-se que, não obstante as limitações dessas ferramentas, é necessário explorá-las mediante abordagem com tarefas pedagógicas, nas quais o foco esteja no significado e não na forma, as competências linguístico-discursivas transitórias dos aprendizes sejam respeitadas e diferentes gêneros discursivos sejam trabalhados. Palavras-chave: chatbots - ensino-aprendizagem - LE/SL - tarefas.
\end{abstract}

\begin{abstract}
This article presents a review of literature on the use of AIML conversational agents (chatbots) as support for foreign/second language teaching and learning. It is argued that, despite the limitations of these tools, it is necessary to exploit them through pedagogical tasks in which the focus is on meaning and not form, the learners' transitional linguistic-discursive competences are taken into account, and different genres are considered.

Keywords: chatbots - second/foreign language teaching and learning - tasks.
\end{abstract}

\section{INTRODUÇÃO}

A aprendizagem de outra língua, além daquela adquirida naturalmente na infância, costuma ser afetada por fatores contextuais e psicológicos que podem retardar ou até impedir o desenvolvimento das competências linguística e discursiva dos aprendizes. Aprendizes de língua estrangeira (LE) costumam enfrentar o problema de acesso limitado ao insumo da língua-alvo de sua aprendizagem devido a fatores como as reduzidas cargashorárias das escolas de idiomas, a dificuldade de acesso a falantes nativos com quem possam interagir e o alto custo de materiais paradidáticos, entre outros.

Aprendizes de língua segunda língua (SL), por sua vez, embora vivam imersos em contextos de uso cotidiano de sua língua-alvo, podem sofrer bloqueios psicológicos oriundos do temor de terem sua competência em desenvolvimento mal avaliada por 
usuários nativos ou até mesmo ter pouco acesso a esses usuários por conta de processos de segregação.

A popularização da Internet, a partir do fim da década de 1990, potencialmente ampliou, para aprendizes de LE/SL, o acesso a uma ampla gama de gêneros discursivos devido à criação de milhares de sites de revistas e jornais. Mais recentemente, também foi ampliado o acesso a conteúdos em áudio e vídeo produzidos por e para usuários nativos de diversos idiomas.

Infelizmente, o acesso a conteúdos multimídia da Internet costuma ter o preço de uma conexão em banda larga, serviço pelo qual podem ser cobradas tarifas altas. Além disso, nem todo conteúdo disponível na rede mundial é confiável ou se presta à exploração imediata por aprendizes de LE/SL sem uma prévia adaptação pedagógica.

Não obstante a existência desses limitadores, há outros serviços associados à Internet que podem ser explorados no auxílio à aprendizagem de LE/SL, mas que não demandam do usuário a assinatura de um serviço de banda larga, como é o caso das redes sociais (Orkut, Facebook, Twitter), dos aplicativos de comunicação instantânea (MSN, Skype), das tradicionais salas de chat, das listas de e-mail e dos fóruns.

Outro serviço de interesse são os agentes conversacionais, também conhecidos como chatterbots ou chatbots ${ }^{1}$, cuja análise é objetivo deste trabalho. Mais especificamente, analiso, com base na literatura da área, as vantagens e desvantagens de chatbots programados na linguagem AIML, bem como uma forma de explorá-los com benefícios para os processos de ensino e aprendizagem de LE/SL.

\section{CHATBOTS}

O termo chatbot foi proposto em 1994 por Michael Maulding para identificar os programas de computador que permitem a interação (conversação) com usuários humanos por meio de linguagem natural (Abu Shawar \& Atwell, 2007:31). A idéia de desenvolver um programa de computador capaz de interagir em linguagem natural a ponto de enganar seu interlocutor humano e fazê-lo acreditar que conversa com outro humano surgiu a partir da publicação, em 1950, do artigo “Computing Machinery and Intelligence” pelo

\footnotetext{
${ }^{1} \mathrm{O}$ termo chatbot será doravante empregado de forma exclusiva neste trabalho.
} 
matemático Alan Turing (1912-1954). Chatbots são empregados em serviços de atendimento a clientes, ferramentas de comunicação instantânea, jogos e até mesmo na educação (Abu Shawar \& Atwell, 2007).

\section{ELIZA}

A primeira geração de chatbots surgiu com o ELIZA, criado em 1966 pelo cientista da computação do MIT Joseph Weizenbaum para simular o diálogo com um analista rogeriano. Esse chatbot funcionava pela detecção de palavras-chave no input do usuário humano e a produção de respostas a partir da aplicação de transformações baseadas no contexto linguístico imediato, o que Weizenbaum denominou scripts (Heller et al., 2005). ELIZA, como muitos chatbots que o sucederam, era incapaz de atribuir sentido às palavras processadas (Abu Shawar \& Atwell, 2007:37), mas, para surpresa de seu próprio criador, era capaz de enganar seus interlocutores mediante estratégias simples como a inversão de pronomes e blocos genéricos, que funcionavam como orações principais em grande número de contextos (Wallace, 2009:184-185).

Essas estratégias são destacadas no trecho de diálogo abaixo (Rich \& Knight, 1983):

USER: Well, my boyfriend made me come here.

ELIZA: Your boyfriend made you come here.

USER: He says I'm depressed much of the time.

ELIZA: I am sorry to hear that you are depressed. ${ }^{2}$

No exemplo, a primeira elocução do usuário continha o sintagma nominal meu namorado. ELIZA simplesmente repetiu essa elocução, invertendo o referente pronominal. Esse tipo de processamento imita o procedimento de um analista rogeriano, criando a ilusão de que o chatbot presta atenção ao que seu interlocutor humano diz.

Efeito semelhante é obtido pelo recurso a blocos prontos que podem ser encaixados como orações principais em quaisquer fragmentos do insumo do interlocutor humano. No exemplo, vemos que, pela segunda, vez ELIZA repete a elocução original efetuando a inversão pronominal (“eu estou deprimida” > “você está deprimida”), mas agora insere a

\footnotetext{
${ }^{2}$ Tradução: Humano: Bem, meu namorado me obrigou a vir aqui. ELIZA: Seu namorado obrigou você a vir aqui. Humano: Ele diz que eu estou sempre deprimida. ELIZA: Lamento saber que você está deprimida.
} 
elocução resultante dentro de um bloco pronto ("Lamento saber que -”). O uso do verbo lamentar, que expressa sentimento, reforça a ilusão de que o chatbot se envolve no discurso de seu interlocutor.

\section{A.L.I.C.E.}

Segundo seu criador, o doutor em ciência da computação Richard Wallace, A.L.I.C.E. - acrônimo de Artificial Linguistic Internet Computer Entity - é um robô de conversação em linguagem natural que se aproxima de seu antecessor ELIZA por também ser estruturado em uma arquitetura de estímulo-resposta (Wallace, 2009:182).

A superioridade de A.L.I.C.E., entretanto, estaria apoiada no número muito maior de unidades de conhecimento (mais de 40.000) em relação ao apresentado por ELIZA (cerca de 200) e na possibilidade de expansão desse conhecimento pelo trabalho do botmaster, técnico humano que monitora as interações de A.L.I.C.E. para ampliar o número de unidades criando novos arquivos na linguagem AIML (Teixeira \& Menezes, 2003: 457; Wallace, 2009:182).

O ‘cérebro’ dos chatbots dessa família é constituído de aproximadamente 41.000 elementos, chamados categorias, programados em AIML, que são processados por um programa que armazena padrões em um dispositivo capaz de associar estímulos a respostas (Wallace, 2009:182). Dessa forma, esses chatbots apenas reconhecem padrões de frases e palavras, e os associam a respostas adequadas ao contexto (Heller et al., 2005: 3914), mas, à semelhança de ELIZA, não são capazes de atribuir sentidos à linguagem processada (Abu Shawar \& Atwell, 2007:39).

O exemplo abaixo, extraído de um corpus coletado por este pesquisador, exibe algumas características do funcionamento de A.L.I.C.E.:

USER: Hi

BOT: Hello there.

USER: How doing?

BOT: How What do you do in your spare time?

USER: $\underline{\text { Read }}$

BOT: What kind of books do you like?

USER: Sci fi 
BOT: I like to read history and biography myself, and other nonfiction.

\section{USER: Are you a writer}

BOT: Am I a writer? I don't know.

USER: Maybe so

BOT: Yippee!

USER: Have to go now

BOT: Bye for now.

USER: Bye

BOT: Adios. ${ }^{3}$

Vemos que o uso de Read (Leitura) pelo interlocutor humano funcionou como uma palavra-chave, permitindo que a interação durasse alguns turnos, com manutenção da unidade temática, ao ativar a pergunta "What kind of books do you like" e a declaração "I like to read history and biography myself”.

O chatbot também não se limitou apenas a repetir a elocução do humano (“Am I a writer”), à semelhança de ELIZA, mas acrescentou uma elocução contextualmente relevante ("I like to read history and biography myself, and other nonfiction”).

A.L.I.C.E. é o chatbot mais bem-sucedido de todos os tempos, pois não só venceu três edições (2000, 2001 e 2004) do Prêmio Loebner, competição que escolhe o chatbot capaz de manter diálogos mais semelhantes aos humanos, mas também originou uma grande família de chatbots (Heller et al., 2005).

\section{AIML}

A Artificial Intelligence Markup Language (AIML) é uma linguagem de marcação, derivada da $\mathrm{XML}^{4}$, desenvolvida por Richard Wallace e por uma comunidade mundial de voluntários a partir de 1995 (Wallace, 2009:191). Os elementos mais importantes da AIML

\footnotetext{
${ }^{3}$ Tradução: Humano: Oi. Chatbot: Olá. Humano: O que fazendo? Chatbot: Como O que você faz no seu tempo livre? Humano: Leitura Chatbot: De que gênero? Humano: Ficção Científica Chatbot:Já eu gosto de História e Biografias, e outros tipos de não-ficção. Humano: Você é escritor (?) Chatbot: Eu sou escritor? Não sei. Humano: Talvez seja Chatbot: Oba! Humano: Preciso sair agora Chatbot: Até logo. Humano: Bye Chatbot: Adios.

${ }^{4}$ Acrônimo de eXtensible Markup Language, padrão de descrição de conteúdos na World Wide Web em termos do tipo de dado que é descrito por meio de tags (Lowell Thing, 2003).
} 
são as tags (marcações) category (categoria), pattern (padrão) e template (modelo) (Wallace, 2009:193).

Categoria é a unidade de conhecimento básica na AIML. Ela é composta de uma pergunta ou elocução que serve de padrão de entrada ( $<$ pattern $><$ pattern $>^{5}$ ) e, pelo menos, um modelo de resposta (<template $></$ template $>$ ).

O padrão é composto formalmente de texto (letras e números), espaços e os curingas _ $\mathrm{e}^{*}$, que podem funcionar como texto (ibid). O modelo é simplesmente composto de texto sem formatação, podendo também conter códigos a serem executados pelo programa processador da AIML (ibid).

Um exemplo de implementação da AIML é fornecido por Michelle Leonhardt (Leonhardt et al., 2003:6):

Código AIML:

$<$ aiml $>^{6}$

$<$ category $>$

$<$ pattern $>$ OI $*<$ pattern $>$

$<$ template $>$ Oi. Tudo bom? $</$ template $>$

$</$ aiml $>$

$</$ category $>$

Diálogos possíveis:

Humano: Oi! / Oi, (Pedro).

Chatbot: Oi. Tudo bom?

Para permitir que o chatbot responda de forma diferente cada vez que o mesmo padrão for apresentado, criando assim a ilusão de certa espontaneidade, pode-se recorrer às marcações $<$ random $><$ /random $>$ e $<$ li $></$ li $>^{7}$, como demonstrado e exemplificado a seguir.

$<$ aiml $>$

$<$ category $>$

$<$ pattern $>\mathrm{OI}<$ pattern $>$

$<$ template $>$

\footnotetext{
${ }^{5}$ Toda marcação precisa ser aberta (p.ex. < pattern>) e fechada (p.ex. </pattern>), daí a notação em pares.

${ }^{6} \mathrm{~A}$ tag $<$ aiml $></$ aiml $>$ delimita todas as marcações dentro de um arquivo programado nessa linguagem.

${ }^{7}$ A marcação <random $><$ random $>$ inicia um processo de seleção automática a partir de opções introduzidas pela marcação $<$ li $></$ li. $>$
} 


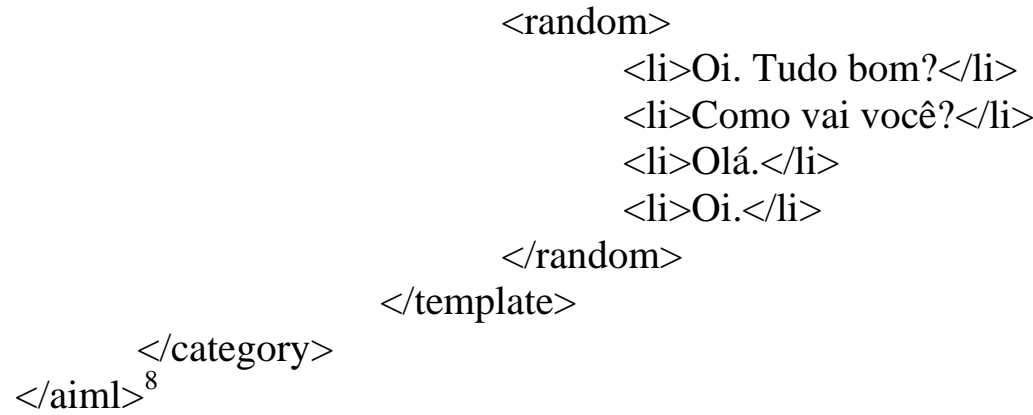

Diálogo correspondente:

\section{Humano: Oi!}

Chatbot: Oi. Tudo bom? / Como vai você? / Olá. / Oi.

No código anterior, as diversas opções de modelo estão associadas ao mesmo padrão original (OI) e são selecionadas aleatoriamente cada vez que o padrão for apresentado como insumo pelo interlocutor humano. Para permitir que as opções de modelo sejam associadas aleatoriamente a outro padrão (p.ex. OLA), pode-se recorrer à marcação $<$ srai $><$ /srai $>$, demonstrada e exemplificada a seguir.

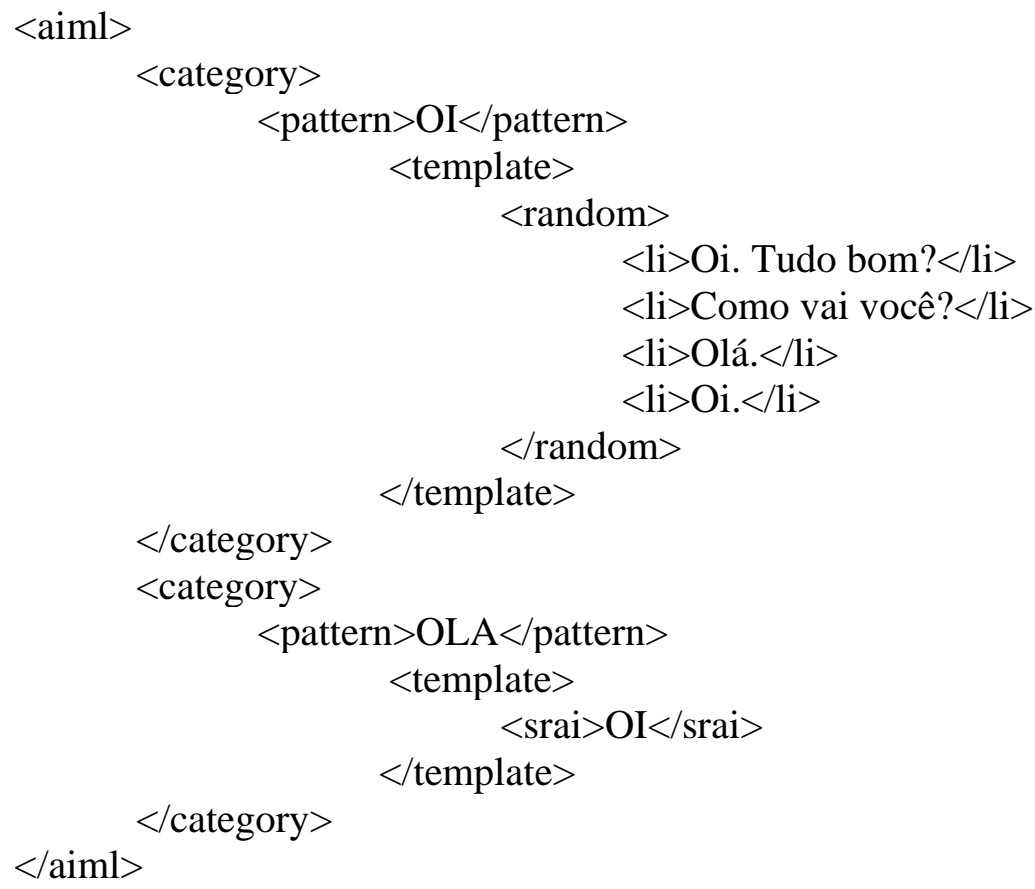

\footnotetext{
${ }^{8}$ Este exemplo e o seguinte são citados de Ferreira \& Uchôa (2006).
} 
Diálogos possíveis:

Humano: Oi! / Olá!

Chatbot: Oi. Tudo bom? / Como vai você? / Olá. / Oi.

A codificação de categorias de conhecimento (blocos de padrões e modelos) em AIML é trabalhosa, e Richard Wallace afirma que “a arte de programar em AIML está em compor respostas padronizadas que sejam breves, concisas, interessantes, gramaticalmente corretas e, por vezes, bem-humoradas, que funcionem com uma grande variedade de insumos” (Wallace, 2009:186).

\section{CHATBOTS NO ENSINO-APRENDIZAGEM DE LE/SL}

Nesta seção apresento alguns estudos que foram realizados com o intuito de avaliar o uso de chatbots nos processos de ensino e aprendizagem de LE/SL.

Jia (2003) descreve um estudo, realizado em universidades e faculdades da China, com cerca de 1250 aprendizes de língua inglesa que tiveram analisados seus registros de interação com um chatbot AIML durante três meses. Foram avaliados fatores como a frequência de uso do chatbot pelos estudantes, a duração das interações mantidas com a ferramenta e comentários de cunho avaliativo feitos pelos estudantes durante as interações. Os resultados desse estudo revelam que a maioria dos estudantes visitava o chatbot apenas uma vez e não retornava. Além disso, as interações eram frequentemente breves, e os comentários de cunho negativo predominavam, com os estudantes geralmente dirigindo ofensas à ferramenta em função de suas limitações.

Os elogios feitos, entretanto, ressaltavam que a ferramenta era vantajosa porque podia interagir com muitas pessoas simultaneamente e jamais se cansava no processo.

O pesquisador conclui que o chatbot AIML não é adequado para o propósito de ensinar uma língua estrangeira porque não é, de fato, capaz de compreender a linguagem, podendo, inclusive, expor o aprendiz a elocuções gramaticalmente incorretas ${ }^{9}$. Além disso,

\footnotetext{
${ }^{9}$ Segundo exemplo citado no artigo, a frase “Are you a come?”, gramaticalmente incorreta, poderia ser aprovada pelo processador AIML que contivesse o padrão <pattern>ARE YOU A *</pattern>.
} 
o modelo estímulo-resposta de um chatbot AIML não dá conta do potencial de inovação linguística descrito por Chomsky (Marcondes, 2009.)

Ferreira \& Uchôa (2006) apresentam a proposta de desenvolvimento de um chatbot AIML para auxílio ao ensino de espanhol como LE no Brasil. Eles justificam a escolha desse tipo de chatbot pela facilidade de desenvolvimento em AIML, mas também destacam aspectos positivos mais gerais do uso de chatbots tais como o controle de informações disponíveis aos aprendizes, evitando-se os riscos apresentados por outras fontes de insumo disponíveis na Web; a possibilidade de diversificação de temas e o potencial de motivação dos aprendizes mais tímidos. Os autores, entretanto, declaram reconhecer as limitações como a incapacidade do programa para compreensão da linguagem e para lidar com a infinidade de elocuções que podem ser produzidas por um interlocutor humano.

Fryer \& Carpenter (2006) ressaltam que: além de serem baratos e acessíveis, chatbots podem reduzir a ansiedade dos aprendizes, permitindo assim que usem a língua-alvo sem bloqueios psicológicos; eles são capazes de repetir conteúdos sob demanda dos aprendizes e sem apresentar exaustão; permitem a ênfase em conteúdos (técnicos ou tabu) normalmente não abordados em escolas de idioma; podem oferecer correção gramatical e ortográfica se programados para isso; e permitem que o aprendiz reveja o registro de sua interação e o analise de modo a perceber ou ter apontados aspectos de sua competência linguística que ainda demandem aperfeiçoamento. Os autores, entretanto, reconhecem que não havia, no momento de produção de seu texto, um chatbot elaborado com a finalidade de auxiliar aprendizes de línguas estrangeiras e concluem que as ferramentas genéricas existentes poderiam ser úteis para aprendizes de nível mais avançado. Eles também afirmam que chatbots para tarefas mais específicas ${ }^{10}$ precisariam ser criados para auxiliar aprendizes iniciantes.

Coniam (2008a) relata um estudo feito para avaliar a precisão linguística de alguns chatbots disponíveis na época. Foram avaliados vários aspectos do desempenho desses chatbots: no nível lexical, o foco foi posto na amplitude vocabular, na capacidade de detectar erros de ortografia e de uso de classes gramaticais; nos níveis sintático e semântico, o foco foi posto na capacidade de processar elocuções simples e sem

\footnotetext{
${ }^{10}$ Como o Freudbot, descrito em Heller et al. (2005), que foi criado para responder perguntas de alunos de psicologia de uma universidade canadense como se fosse Sigmund Freud, o pai da psicanálise.
} 
ambiguidades, de responder perguntas formuladas com erros, de lidar com elocuções contendo itens lexicais em ordem incorreta e de manter a lógica pronominal em elocuções reescritas; no nível suprassentencial e discursivo, foram avaliadas a capacidade de processar elocuções contendo mais de uma oração, de interpretar conhecimento de mundo e de lidar com mudanças de tópico discursivo. O resultado desse estudo aprofundado indica que os chatbots analisados são capazes de lidar relativamente bem com elocuções contendo oração simples, organizadas de forma direta, abordando assuntos cotidianos, sem laços coesivos com orações prévias e redigidas sem erros de ortografia. Em resumo, eles apresentam uma limitação importante no processamento de insumo espontaneamente produzido, que muito provavelmente não possui essas características formais.

Outro trabalho desse autor (Coniam, 2008b) analisa os mesmos chatbots, mas desloca o foco para aspectos como a interface com o usuário, o tempo de resposta e a usabilidade. A conclusão é que a proposta de uma conversação simulada convincente traz exigências significativas para que esses sistemas sejam explorados nos processos de ensino e aprendizagem de LE/SL. Em termos gerais, eles precisam apresentar interfaces simples de usar e sem excesso de recursos e informações e, quando incorporam voz sintetizada, precisam garantir boa qualidade em entonação, ritmo e padrões de tonicidade.

Defendo que esses resultados não sejam interpretados de forma definitiva, pois, além de a quantidade de pesquisas ser reduzida, elas avaliaram chatbots apenas pelo que não conseguiam fazer, em alguns momentos deslocando para segundo plano o que eles potencialmente ofereceriam sob condições pedagogicamente mais bem elaboradas.

\section{TAREFAS PEDAGÓGICAS COM USO DE CHATBOTS}

Em lugar de simplesmente explorar uma capacidade que os chatbots não têm dialogar com humanos de forma absolutamente espontânea - pode ser mais produtivo assimilá-los a uma abordagem de ensino na qual tarefas pedagógicas demandem uso da língua-alvo com propósitos específicos, ou seja, nas quais o diálogo na língua-alvo seja um meio e não um fim em si mesmo ${ }^{11}$.

\footnotetext{
${ }^{11}$ Não está no escopo deste trabalho discutir as vantagens da abordagem baseada em tarefas. Apenas pretendo apresentar essa abordagem como uma alternativa para apropriação dos chatbots nos processos de ensinoaprendizagem de línguas.
} 
Adoto aqui a perspectiva de David Nunan (1991:10), para quem a tarefa é uma “atividade de estudo que envolve o aprendiz nos processos de compreensão, manipulação, produção ou interação na língua-alvo, enquanto põe sua atenção no significado e não na forma” ${ }^{12}$. Uma tarefa é composta dos seguintes fatores, descritos por Nunan (1991:47-78):

- Objetivo: o que se pretende obter com a tarefa em termos comunicativos, afetivos ou cognitivos (p.ex.: capacitar o aprendiz para o intercâmbio de informações por meio do diálogo com chatbot);

- Insumo: informação na língua-alvo que será processada pelo aprendiz (p.ex.: formulário a ser preenchido com as respostas do chatbot, registro da interação realizada para autoavaliação pelo aprendiz);

- Atividades: ações que o aprendiz executará no processamento do insumo e que podem apresentar ênfase no ensaio ou na execução real, na fluência ou na precisão linguística (p.ex.: leitura do formulário, produção de perguntas para entrevista com o chatbot, elaboração de texto a partir das respostas fornecidas por ele);

- Papel do professor: funções que o professor exercerá durante a execução da tarefa pelo aprendiz (p.ex.: monitor que observa o desempenho do aprendiz e dá apoio quando necessário);

- Papel do aprendiz: funções que o aprendiz exercerá durante a execução da tarefa (p.ex.: entrevistador do chatbot, analista de linguagem, redator);

- Contexto: local e tipo de organização assumidos na realização da tarefa (p.ex.: arranjo em semicírculo no laboratório de informática durante a produção de perguntas, interação direta e individual com o chatbot no laboratório de informática).

Para dar conta das necessidades - e competências transitórias - dos aprendizes, em lugar de simplificar o insumo linguístico fornecido pelo chatbot, pode-se optar pela variação no nível de dificuldade presumido das atividades propostas a eles, como sugere

\footnotetext{
12 Minha tradução do original: “A piece of classroom work involving learners in comprehension, manipulation, production or interaction in the target language, while their attention is focused on meaning rather than form".
} 
Nunan (1991: 104). ${ }^{13}$ Esse autor também sugere critérios para organização de atividades de acordo com seu nível de complexidade para os níveis de competência linguística Iniciante, Pré-Intermediário e Intermediário (op.cit:113-115).

No Apêndice, apresento um exemplo de tarefa complexa com uso de chatbot que oferece aos aprendizes de LE/SL oportunidades tanto para estudo aprofundado de aspectos linguísticos e textuais quando para aprendizagem e uso de diferentes gêneros discursivos.

\section{CONCLUSÃO}

Chatbots AIML são criados para gerar a ilusão de que a máquina é capaz de manter um diálogo espontâneo e prolongado com um interlocutor humano. Entretanto, algumas pesquisas sugerem que essas ferramentas não são capazes de substituir usuários linguisticamente competentes ou atuar como professores ou tutores no ensinoaprendizagem de LE/SL.

De qualquer forma, não se deve desprezar as vantagens dessa tecnologia barata e segura, que pode oferecer a aprendizes de LE/SL acesso a insumo da língua-alvo em contexto lúdico e sem a pressão psicológica que costuma caracterizar a interação com usuários nativos dessa língua ou com colegas e professores na sala de aula.

A solução pode estar na assimilação dos chatbots a uma abordagem de ensino na qual:

(a) a língua-alvo possa ser empregada com foco no significado e não apenas na forma;

(b) a competência linguístico-discursiva transitória do aprendiz é respeitada;

(c) as atividades pedagógicas ofereçam ao aprendiz a oportunidade de conhecer e empregar novos gêneros discursivos.

Essa abordagem - baseada em tarefas pedagógicas - será alvo de estudos mais aprofundados em meu projeto de pesquisa.

\footnotetext{
${ }^{13}$ No original: "In recent years, with the increasing use of authentic texts, there has been a tendency to control difficulty not by simplifying the input to which learners are exposed, but by varying the difficulty of the activities which learners are expected to carry out.”
} 


\section{REFERÊNCIAS}

Abu Shawar, B. \& Atwell, E. (2007). Chatbots: are they really useful? LDV-Forum - Band 22(1), 31-50.

Coniam, D. (2008a). Evaluating the language resources of chatbots for their potential in English as a Second Language. ReCALL, 20(1), 98-116.

Coniam, D. (2008b). An evaluation of chatbots as software aids to learning English as a second language. EuroCALL Review, 13. On-line http://www.eurocalllanguages.org/news/newsletter/13/. Acesso 24 fev. 2010.

Ferreira, L.P. \& Uchôa, J. Q. (2006). Desenvolvimento de um chatbot para auxiliar o ensino de Espanhol como Língua Estrangeira. Bazar: Software e Conhecimento Livres, (1): 1, 21- 32.

Fryer, L. \& Carpenter, R. (2006) Emerging Technologies: Bots as Language Learning Tools. In Language Learning \& Technology, 10(3) , 8-14.

Heller, B. et al. (2005). Freudbot: An Investigation of Chatbot Technology in Distance Education. In P. Kommers \& G. Richards (Eds.), Proceedings of World Conference on Educational Multimedia, Hypermedia and Telecommunications. Chesapeake, VA: AACE, 3913-3918.

Jia, J. (2003). The Study of the Application of a Keywords-based Chatbot System on the Teaching of Foreign Languages - Institute for Interdisciplinary Informatics - University of Augsburg - Germany

Leonhardt, M. D. et al. (2003). ELEKTRA: Um Chatterbot para Uso em Ambiente Educacional. In Ciclo de Palestras Novas Tecnologias na Educação. V. $1 \mathrm{~N}^{\mathrm{o}}$ 2, Porto Alegre, RS.

Marcondes, D. (2009). Chomsky: Linguagem e pensamento. In Textos básicos de linguagem: de Platão a Foucault. Rio de Janeiro: Jorge Zahar Ed., 110-114.

Nunan, D. (1989) Designing Tasks for the Communicative Classroom, Cambridge: Cambridge University Press.

Rich, E., \& Knight, K. (1983). Artificial Intelligence. (2nd ed.), McGraw-Hill.

Teixeira S. \& Menezes, C. S. (2003). Facilitando o uso de Ambientes Virtuais através de Agentes de Conversação. XIV Simpósio Brasileiro de Informática na Educação - SBIE 2003, Rio de Janeiro, RJ, Brasil, 483-492. 
Wallace, R.S. (2009). “The Anatomy of A.L.I.C.E”. In Epstein, R. et al. (Eds.) Parsing the Turing Test: Philosophical and Methodological Issues in the Quest for the Thinking Computer. Springer, 180-210.

XML. In Lowell Thing. (2003) Dicionário de tecnologia. São Paulo: Futura, 977-978.

\section{APÊNDICE - Entrevista com um Robô-Personalidade ${ }^{14}$}

Objetivos: Intercâmbio de informações e produção textual.

Apresentação: Nesta tarefa, você conversará com um chatbot, um programa de computador capaz de manter um diálogo quase natural com o ser humano. Será uma excelente oportunidade para você praticar seus conhecimentos da língua que está estudando, mas também para conhecer melhor a personalidade que esse chatbot se propõe a representar.

Etapa 1: Antes de entrevistar [NOME DA PERSONALIDADE], você precisará elaborar o roteiro da entrevista, preparando antecipadamente as perguntas que deseja fazer ao entrevistado. Eis algumas dicas:

a) Pense em quais aspectos da vida dessa personalidade interessam a você e às pessoas que lerão o resultado de sua entrevista. Será que interessa saber sobre a infância dessa 'pessoa'? Ou sobre os motivos que a levaram à atividade que desempenha hoje? Proponha no mínimo 4 grandes blocos de interesse (exemplo: Infância - Juventude - Vida Familiar - Planos para o Futuro) dentro dos quais você inserirá as perguntas. Nesta atividade, você poderá contar com a ajuda dos colegas e do professor.

b) Para cada bloco, elabore até cinco perguntas. Depois que as tiver escrito, apresente ao professor para que ele avalie se há problemas que precisem ser corrigidos antes da entrevista. Reescreva as perguntas indicadas pelo professor, mas tente observar em que aspectos você errou mais. Se necessário, faça uma revisão gramatical. O professor poderá orientar a esse respeito.

Etapa 2: Agora você poderá entrevistar o [NOME DA PERSONALIDADE]-robô usando as perguntas elaboradas na Etapa 1. É importante que você guarde um registro completo dessa entrevista, portanto verifique com seu professor se o robô produz esse registro automaticamente.

Etapa 3: Dedique-se às seguintes atividades:

a) Leia o registro da entrevista e destaque nas respostas do robô as informações novas, isto é, o que você realmente não sabia sobre seu entrevistado e chamou sua atenção. Essa informação seria de interesse de outras pessoas também? Discuta suas descobertas com outros colegas da turma.

\footnotetext{
${ }^{14}$ Alguns chatbots AIML receberam bases de conhecimentos de personalidades como o Capitão James T. Kirk (http://bit.ly/9j6pf6), da série de TV Jornada nas Estrelas, e Sigmund Freud (http://bit.ly/b2U7wq).
} 
b) Nas respostas, o robô empregou alguma palavra ou forma gramatical que você desconhecia? Você consegue deduzir seu significado? Peça ajuda aos colegas ou ao professor ou, ainda, consulte um dicionário ou gramática. Anote suas descobertas.

c) Aproveite a informação nova e interessante sobre [NOME DA PERSONALIDADE] e produza um dos seguintes textos, a sua escolha:

a. Um verbete curto para uma enciclopédia descrevendo essa personalidade.

b. Uma matéria para o jornal da escola em que você falará sobre essa personalidade, destacando os fatos provavelmente menos conhecidos da vida dela.

i. Se necessário, peça ao professor que oriente nas formas possíveis para organizar seu texto;

ii. Leia verbetes ou matérias e tente observar como o texto foi organizado. Avalie se você precisará seguir a mesma organização ou se poderá fazer algo diferente.

\section{O AUTOR}

José Paulo de Araújo é doutorando do Programa Interdisciplinar de Pós-Graduação em Linguística Aplicada da Universidade Federal do Rio de Janeiro, no qual está associado ao núcleo de pesquisa Linguagem, Educação e Tecnologia - LingNet. Sua pesquisa investiga novas tecnologias da informação e comunicação (NTICs) que têm potencial na facilitação dos processos de ensino e aprendizagem.

e-mail: josepaulo@ufrj.br 